\title{
Possible triggered star formation associated with SNR G59.5+0.1
}

\author{
Jin-Long $\mathrm{Xu}$ and Jun-Jie Wang \\ National Astronomical Observatories, Chinese Academy of Sciences, \\ 100012 Beijing, China; email: xuj1@bao.ac.cn
}

\begin{abstract}
We perform the submillimeter/millimeter observations in CO lines toward the southeast of SNR G59.5+0.1 with the KOSMA 3m-Telescope, which fully covers the open cluster NGC 6823. Three molecular clumps are identified in the CO molecular arc, each clump show outflow in motion. The age of the SNR is $8.6 \times 10^{4} \mathrm{yr}$. The number of selected YSOs are significantly enhanced in the interacting regions, indicating the presence of some recently formed stars.
\end{abstract}

Keywords. ISM: clouds — ISM: individual (G59.5+0.1) — ISM: molecules — stars: formation

Massive stars can have a significant impact on the morphology and chemical evolution of the surrounding ISM by means of ultraviolet (UV) radiation, stellar winds, and supernova explosions. SNR G59.5+0.1 is at a distance of 2.12.3 kpc (Taylor et al. 1992).

The distribution of redshifted and blueshifted velocity components in Panel b provides us with further evidence of the bipolar outflow in each clump. The average dynamical timescale of the outflows is $10^{4} \mathrm{yr}$. It may provide us a direct evidence for star formation triggered by SNR. To further look for primary tracers of star formation activity around G59.5+0.1, we select 176 Class I sources and 449 Class II sources. The asymmetric distribution of YSOs in Panel c indicates the presence of some recently formed star.
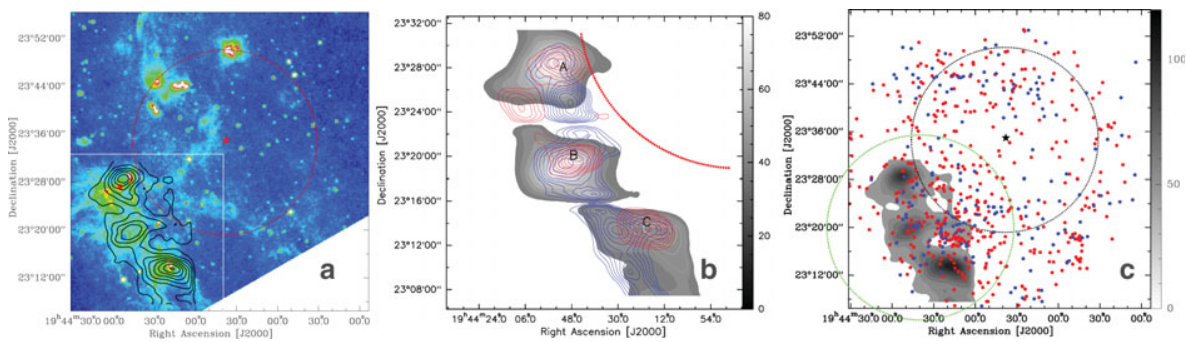

Figure 1. Panel a: ${ }^{12} \mathrm{CO} J=2-1$ intensity map (black contours) overlaid on the mid-infrared 8.28- $\mu$ m MSX emission map (color scale). The red star represents the center of SNR G59.5+0.1 and its extent is outlined by a red dashed circle. Panel b: The velocity-integrated intensity maps of ${ }^{12} \mathrm{CO} J=2-1$ outflows (red and blue contours) overlaid with the ${ }^{12} \mathrm{CO} J=2-1$ emission of each clump (gray scale). Panel c: The Class I sources and Class II sources are labeled as the blue dots and the red dots. The green dashed circles and the open cluster NGC6823 (a color version of this figure is available online).

\section{Acknowledgements}

We thank Dr. Martin Miller and Ms. Ni-Mei Chen for her help during the observations.

\section{References}

Taylor, A. R., Wallace, B. J., \& Goss, W. M., 1992, AJ, 103, 931

Xu, J. L. \& Wang, J. J. 2012, A\&A, 543, 24 